\title{
Usability Assessment of ASIBOT: a Portable Robot to Aid Spinal Cord
}

\section{Injury Patients}

Authors: Alberto Jardón ${ }^{1}$, Ángel M. Gil ${ }^{2}$, Ana I. De la Peña ${ }^{2}$, Concepción A. Monje ${ }^{1}$, Carlos Balaguer ${ }^{1}$
${ }^{1}$ RoboticsLab, Systems Engineering and Automation Department, University Carlos III of Madrid, Spain.
${ }^{2}$ Biomechanics and Technical Aids Unit, Spinal Cord Injury National Hospital, SESCAM, Toledo, Spain.

Correspondence address:

Alberto Jardón Huete, RoboticsLab, Systems Engineering and Automation Department, University Carlos III of Madrid, Spain.

Avenida Universidad N³0, 28911 Leganés, Madrid

Telephone: +34916246242

Fax: +34916249430

E-mail: ajardon@ing.uc3m.es

Keywords: Assistive Technology, Functional Evaluation, Rehabilitation Engineering, Robotics, Technical Aids, Human Factor. 


\begin{abstract}
The usability concept refers to aspects related to the use of products that are closely linked to the user's degree of satisfaction. Our goal is to present a functional evaluation methodology for assessing the usability of sophisticated technical aids, such as a portable robot for helping disabled patients with severe spinal cord injuries. The specific manipulator used for this task is ASIBOT, a personal assistance robot totally developed by RoboticsLab at the University Carlos III of Madrid. Our purpose is also to improve some aspects of the manipulator according to the user's perception. For our case study a population of six spinal cord injury patients is considered. These patients have been suffering spinal cord injuries for a period of time longer than one year before the tests are carried out. The methodology followed for the information gathering is based on the Quebec User Evaluation of Satisfaction with assistive Technology (QUEST). Different daily functions, such as drinking, brushing one's teeth, and washing one's face, are considered to assess the user's perception when using ASIBOT as a technical aid. The human factor in this procedure is the main base to establish the specific needs and tools to make the end product more suitable and usable.
\end{abstract}




\section{INTRODUCTION}

In the disability sector, there is a steady stream of new products that interact with the users at a higher or lower degree in order to meet their everyday needs. In addition to making a technical assessment of these products, it is also appropriate to implement a functional evaluation procedure designed by experts where the users' opinion and degree of satisfaction are taken into account [1] [2] [3].

The usability concept is closely linked to the user's degree of satisfaction with the product. This concept is used to measure how useful the product and the system settings are for the user to achieve specific goals efficiently, effectively, and satisfactorily in a specific context [4]. The product design process entails numerous activities, some of them not strictly related to the usability, that must be taken into account and incorporated into the product. These aspects should not be left for further stages: the sooner the functional and ergonomic factors are included, the faster, cheaper, and more effective the design process will become.

A standard evaluation of any product includes an analysis of the planned functions. Therefore, there are several testing procedures and/or evaluation standards that measure how well a product performs its functions in terms of technical quality regulations which evaluate hardness, robustness, and safety. These standards and procedures are related to the characteristics of the technical aid itself or, at most, to the user's mechanical relationship with the product. Considerations of this type are usually part of the process prior to implementing information and communication technologies in the market.

However, the user's satisfaction concerning technology is conditional on the emotional perception that the products evoke to the users. This aspect of designing and implementing a resource is frequently forgotten. Although the market is starting to realize the importance of incorporating strategies that include the final user's technological expectations, these techniques are not always applied or, at least, the way they are is questionable [5] [6] [7].

In this paper, we present our own experience in assessing the usability of ASIBOT [8] [9], a prototype of a personal assistance robot, involving users directly in the whole process to achieve their needs and expectations. The users' perception will be considered in order to improve the prototype and to establish future needs and research goals. Our research has been carried out at the Spinal Cord Injury National Hospital of Toledo, in 
coordination with RoboticsLab at the University Carlos III of Madrid. Participants from both centers have been involved in the development of the questionnaire and data collection, as will be explained next.

\section{OBJECTIVES}

Since it is necessary to establish a link between the users' opinions based on their needs and the product development, our aim is to generate a representative statement of the users' perception in a structured format in order to exert an influence on the improvement of already-existing products and generate adequate design criteria for new ones.

More specifically, the objective of this work is to develop a functional evaluation methodology for assessing the usability of ASIBOT. For this purpose, we will consider a case study with six disabled patients with spinal cord injuries in an intra-hospital environment, simulating an everyday setting for certain basic daily activities. Our aim is to gather structured data from the experiments reflecting the perception of the user when dealing with the robot. These data will serve as a base for improving the design of a new robot prototype.

\section{PROCEDURES}

The methodology followed to assess ASIBOT is based on gathering information in a structured format from the interactions of selected users with the robot. A testing procedure has been created specifying different settings and tasks to be carried out. The information is gathered from each of the users who interact with the technical aid. That information is then analysed. Finally, the results are discussed in order to use them as the base for the design of a new prototype that fulfils the users' needs and expectations.

\section{Description of the users}

The case study considered in this work takes into account users with the following characteristics:

- They are disabled patients with spinal cord injuries.

- They have been suffering spinal cord injuries for a period of time longer than one year before the tests are carried out. 
- They are affected at neurological levels from C4 to C8 (figure 1), which implies that they have mobility limitations in their upper extremities.

- They have spent regular periods of time in their homes before the tests, which gives them a perception of the main difficulties found in their daily activities. Based on their experience in facing numerous dependency problems, they are able to evaluate the functionality of technical aids from a more objective point of view.

No cases with acute injuries are considered. Other exclusion criteria are: epilepsy, mental retardation, uncorrected visual deficiency, or psychiatric problems. We cannot forget that ASIBOT is a prototype to assist patients in specific tasks (not to replace them), but it is the patient who governs the performance of the robot during operation. For this reason, the user must have all the faculties to do so (control and react). That is why these exclusion criteria have been considered.

The case study considers six patients who fulfil the above criteria. Some considerations about the proper number of users to be considered for usability assessments will be given in the Information gathering methodology section of this paper.

Insert figure 1 about here

\section{Description of the personal assistance robot ASIBOT}

The robotic system ASIBOT (figure 2) [8] [9] [10] [11], totally developed by RoboticsLab at the University Carlos III of Madrid, is a significant advance in service robots [9] [12] [13]. All the electronic systems for activation, control, and communications are on board, which makes it the first portable climbing robot able to manipulate things. This means that it can not only be easily moved from one setting to another but also move itself using different connectors. Its only physical connection is a $24 \mathrm{~V}$ DC power supply provided via these connectors, usually located on the wall. The control system given to the user is based on a multi-modal interface implemented in a PDA, which provides an assisted teleoperation system.

Insert figure 2 about here 
ASIBOT has a modular design able to work in a wide variety of settings with a high range of autonomy and mobility, since it can move along the walls, the furniture, or other surfaces with accuracy and reliability via connectors located along the route the robotic arm moves along (figure 3). Once one of the ends of the arm connects to one connector, the other end disconnects and gets free to support the patient (as illustrated in figure 2). This modular design allows readapting the system to the resulting functional demands and to variations in the patient's conditions [8] [9].

Insert figure 3 about here

In addition to its mobility, the fact that it is anchored to a single point allows it to manipulate tools attached to its free end, which has actuators controlled by the system and/or the user [14] [15]. This is of great interest for people with motor disabilities in their upper extremities, since it allows them to bring objects closer and pick up and move utensils for everyday activities to a nearer working area [16]. It is important to remark that all the operations are carried out in safety conditions, since the final contact with the robot or the utensils is performed at the user's command.

In sum, ASIBOT has an innovative design with all the functionalities to assist the disabled patients described above.

\section{Information gathering methodology}

In order to develop a methodology to assess the user's perception and satisfaction when using ASIBOT as an assistance technical aid, the most relevant bibliography related to this subject has been reviewed [5] [6].

Our design approach in this line aims the introduction of the usability concept for specific situations, that is, according to the specific user's characteristics and needs. This way, the methodology to evaluate the product must provide information about each individual user by means of consecutive interactions with them, allowing the patients to benefit directly from the assistance and information gathered in their presence [17] [18]. 
For this reason, and taking into account the lack of procedures for the assessment of user's perception and satisfaction, our design is based on the application of evaluation criteria as defined in what is known as "The K.I.U. Test" [19]. The main goals of these techniques refer to identifying the most frequent problems; detecting errors, needs, or requirements; generating design criteria and final user requirements; as well as a global usability assessment of the product, which allows us to identify which aspects need to be modified in the new design. However, the main deficiencies in usability deal with the following criteria: ease of learning how to use it, utility, functionality, ease of use, and user's satisfaction [3] [20] [21].

These deficiencies have been considered in the design of our own questionnaire. The validity of the data and understanding of the questions are verified previously. The questions are analysed to make sure that they fulfil our objectives. Besides, the total time to complete the questionnaire is not more than 30 minutes, in order to prevent fatigue or distraction. Questions that evoke negative stimuli to the user are eliminated and explanations on how to fill in the questionnaire are included.

The questionnaire for the experiments has four parts:

- A first part with seven closed questions, with answers given on a Likert summative five point satisfaction scale (from +2 to -2 ) and focusing on an assessment of the functions or activities suggested for the robot to perform during the tests.

- The second part consists of questions in the former format focusing on the assessment of eight general characteristics of the robot: appearance, dimensions, usability, speed of response, safety, robustness, multi-use functionality, prior training.

- In the third part, three open questions are proposed about the use of the prototype, its utility, and assistance needs of each patient.

- Finally, a general assessment of the technical aid is done through two specific questions with answers in an ordinal scale format from 0 to 10 points.

The tests have been held in a hospital context, carried out in a suitable scenario to accomplish the studied activities. The facilities at the hospital's Occupational Therapy Unit have been used to partially simulate a daily home environment. 
Regarding the number of users for a proper usability assessment, Virzi [22], Nielsen and Landauer [23], and Lewis [24] have published influential articles on the topic of sample size in usability testing. In these articles, the authors presented a mathematical model of problem discovery rates in usability testing. Using the problem discovery rate model, they showed that it was possible to determine the sample size needed to uncover a given proportion of problems in an interface during one test. The authors presented empirical evidence for the models and made several important claims: 1) Most usability problems are detected with the first three to five subjects; 2) Running additional subjects during the same test is unlikely to reveal new information; 3) Return on Investment (ROI) in usability testing is maximized when testing with small groups using an iterative test-anddesign methodology.

Nielsen and Landauer [23] extended Virzi's original findings [22] and reported case studies that supported their claims for needing only small samples for usability tests. They and Lewis [24] identified important assumptions about the use of the formula for estimating problem discovery rates. The problem discovery rate model was recently re-examined in [25] [26].

According to these researches, five is a proper number for usability testing. In our case, five is the number of patients considered, fulfilling the inclusion criteria stated previously. Therefore, we consider that the results presented in this paper are representative using this sample size. In any case, it must be taken into account that these experiments are a first star and more future tests will be performed with the possibility of including more patients.

\section{Experiment procedure}

Prior to the experiments in real settings with ASIBOT, a pilot test is done to assess the user's understanding of: 1) the potentials and limitations of ASIBOT as a functional robotic aid, by means of explanations based on simulations and video-recorded real experiences.

2) several ways of interacting with the Human-Machine Interface (HMI) and its influence in the overall performance. The HMI front-end is shown to the user and explanations are given on how to interact with it. 
A sample of representative videos is used to explain ASIBOT's functional capabilities, e.g. the scene of teeth brushing with ASIBOT shown in figure 4.

Insert figure 4 about here

In our case, a special experimental set-up for the HMI is implemented in a PDA. In the shape of large visualtactile buttons, the different functions to be performed by the robot can be activated through the following modes, ranked from the greatest to the smallest mobility requirements (figure 5):

- $\quad$ Tactile, via user's touch or a pencil.

- Joystick, to choose the functions with a button to validate them.

- $\quad$ Lighted sequence with a selection button: a sequence of options is lighted and different choices for the options can be selected.

- Voice recognition, with different options for selection and activation by voice.

Insert figure 5 about here

In this pilot test, a questionnaire similar to the one proposed to assess the use of the robot has to be completed. The users are asked about each of the interfaces and their ability to handle them without any help, their ease of use, practicality, and how appropriate each interface is given their capabilities.

Finally, two open questions encourage users to give any suggestions or ideas for making control simpler.

After this pilot test, experiments with the robot in real scenarios have to be performed. In order to do so, the users are previously asked to share their a priori opinion about the situations, activities, or tasks where they think the robot assistance would be helpful. In order of importance, they rank those situations where they need personal assistance. According to that information, the environment is set to assess the functionality of the robot in those adverse conditions described by the users. 


\section{EXPERIMENTAL RESULTS}

The experiment procedure has three main parts:

- First stage: Assessment of interfaces and first contact with the robot.

- Second stage: Handling the robot in a scenario.

- Third stage: Gathered data analysis.

The complete procedure and the experimental results are discussed next.

\section{First stage: Assessment of interfaces and first contact with the robot.}

As commented previously, our case study considers six users who meet the inclusion criteria described in the Description of users section of this paper.

In this first stage, the HMI assessment of the robot is done, as described in the Experiment procedure section. After evaluating the different interfaces offered, the following conclusions are drawn from the users' answers:

- Except for one person, they all are capable of HANDLING ON THEIR OWN the different interfaces offered: voice recognition (with different options), joystick, tactile, and lighted sequence with a selection button.

- Regarding EASE OF USE, their selection in order of preference is: tactile, voice recognition, joystick, and lighted sequence.

- The most PRACTICAL interface turns out to be the tactile one, followed by voice and joystick with the same score, and finally the lighted sequence.

- The interfaces based on VOICE recognition and JOYSTICK are preferred by the users regarding APPROPRIATENESS of the interface given their MOBILITY ABILITIES.

After this first face-to-face session with the users, information is obtained via a questionnaire in order to focus the subsequent real experiments with the robot on the most frequently demanded activities. 
In one of the first questions about their main demands for independence, the users state which activities they find more unpleasant and they would like to be able to do without depending on another person, independently of the capability of the robot to accomplish these tasks or not. Getting dressed and washing themselves, in that order, are mentioned by the majority of the users.

They are also asked to rate (in order of importance) four settings or situations proposed where using the robot would be more helpful. The results in order of importance are the following ones:

1. Daily hygiene: washing their face and hands, brushing their teeth, combing their hair, shaving, or using make-up.

2. Lying in bed.

3. In the wheelchair: eating, drinking, bringing objects closer, etc.

4. In the kitchen: opening cupboard doors, moving utensils, etc.

The results show that personal hygiene functions are the most appropriate to test the robot's performance and assess the user's perception more reliably. For this reason, the final assessment is going to be carried out in a bathroom scenario (figures $6 \mathrm{a}$ and $6 \mathrm{~b}$ ). The assessment of available resources for the experiments also leads to our decision to use the bathroom facing the mirror, given the chances it offers to assess a larger number of basic functions in everyday life. We propose three functions to test in this setting: drinking a glass of water, brushing one's teeth, and washing one's face.

Insert figures $6 \mathrm{a}$ and $6 \mathrm{~b}$ about here

\section{Second stage: Handling the robot in a bathroom scenario.}

For the final experiment, the group selected is composed by five of the six users from the previous stage, since one of them cannot attend the experiment session for personal reasons. They are all male and represent each cervical level of injury from $\mathrm{C} 4$ to $\mathrm{C} 8$. The functions of drinking a glass of water, brushing their teeth, and washing their faces with a sponge are assessed. 
Prior to handle these three situations with the real robot, the users have a first real contact with it (real, not through videos or simulations) and have time to learn how it works and how their interaction with the robot will be performed in these specific situations. It is important to remark that the human factor is vital in all this procedure and we must ensure that the users are receptive to the experiment from the very beginning.

After the experiments, and for each of the functions, the patients are first asked seven questions regarding the robot's performance during the activities. They are instructed (figure 7) to assign a response scored on a Likert satisfaction scale from -2 to +2 , where +2 is "completely satisfied", +1 is "satisfied", 0 is "not sure", -1 is "dissatisfied", and -2 is "completely dissatisfied".

Insert figure 7 about here

The global results of each function are shown in tables 1, 2, and 3, respectively. The most relevant data of each of the three functions are discussed next:

- For the DRINKING function, the average score is 0.91 . A score of 1 would mean "satisfied" and 0 would mean "not sure". Therefore, the use of the robot for this function can be defined as CORRECT (table 1).

- For the BRUSHING ONE'S TEETH function, the average score is 0.49. According to the above criteria, the use of the robot for this function can be also defined as CORRECT (table 2).

- $\quad$ For the WASHING ONE'S FACE function, only two users give a response. The average score is -0.86. A score of -1 would mean "dissatisfied" and 0 would mean "not sure". Therefore, the use of the robot for this function CAN BE IMPROVED (table 3). However, the reliability of this result is conditional on the fact that only a small sample of the group participates in the test.

Insert table 1 about here

Insert table 2 about here

Insert table 3 about here 
From these results, we can conclude that the users' perception is that the robot performs the functions of drinking and brushing their teeth properly. However, its performance when washing their face should be improved.

In a second step, the robot characteristics are evaluated by the users, expressing their opinion with the same range of satisfaction scores, from -2 to +2 . The best results are for its robustness (averaged 1), its multi-use functionality (averaged 1), and its speed of response (averaged 0.8). Lower scores are given to its dimensions and appearance, averaged -0.8 and -0.2 , respectively (table 4$)$.

Insert table 4 about here

In a third step, three open questions are made:

- First of all, users are asked what changes they suggest in order to make the robot more useful. The most common suggestions are: a smaller size, voice recognition, the possibility of including additional tasks adding other terminal devices, and more safety conditions as far as the stopping function of the robot is concerned.

- Additional tasks for this robot that might be useful for the users are: cleaning the house, cooking, making or unmaking the bed, folding sheets, dressing, shaving, tasks requiring accuracy, combing their hair, cutting their nails, picking up glasses, opening windows, and opening doors.

- Finally, they are asked again to rank the most unpleasant tasks they would like to be able to do on their own and not depending on someone else, regardless the capability of the robot to perform such tasks. The personal hygiene tasks stand out against moving around, dressing, and picking up or reaching objects.

Finally, regarding the overall assessment of the robot after its use, they are asked two questions: one concerning the independence the robot could provide and the other one about replacing their carer with the robot, freeing that person from doing the work. The average scores in response to these matters are commented next (table 5):

- They would attain greater independence in their everyday lives: an average of 5 out of 10. 
- They would be interested in freeing their carer or relative from having to perform these tasks to help them: 5.8 out of 10

One patient's answer is worthy of note and will be discussed in the Discussion section. Considering the other four users the average is 6.25 and 7.25 out of 10 , respectively.

Insert table 5 about here

\section{Third stage: Gathered data analysis.}

Once the first and second stages are completed and all the data regarding users' perception through the experiments are gathered, the researchers are ready to discuss the results and do the final assessment of the whole procedure, establishing the relations between the level of injury and the final score for each function. The gathered data analysis for the specific experiments presented here is discussed in the following section.

\section{DISCUSSION}

The evaluation of outcomes in assistive technology is perhaps one of the greatest challenges for rehabilitation practitioners, researchers, engineers, and suppliers of technical aids [20] [21]. Like any product used regularly, technical aids must satisfy users' expectations regarding the functions they hope to perform. In the case of products designed for the field of disabled people, this requirement becomes even more important, considering the fact that the functions to be performed are generally essential for personal autonomy [27] [28] [29] [30].

Due to a lack of theoretical knowledge, the satisfaction determiners are vague in the field of assistive technology. The relations between the variables involved in the experience of satisfaction and the assistive technology are generally represented within a linear general framework, inspired by Simon and Patrick [27]. The Quebec User Evaluation of Satisfaction with assistive Technology (QUEST) (6) is also a pioneer scale for satisfaction measurement. It is considered as the most relevant tool in functional assessment for assistive technology. It covers both the device and the service components of assistive technology [18] [29]. 
Although we agree that this instrument is quite useful, we have decided to develop our own questionnaire because QUEST considers many aspects that cannot be taken into account when prototypes which are not in the market are considered. Another problem is the fact that a proposal of modifications is required to improve the functional aspects of the current prototype to develop a new improved one. Therefore, the device must be assessed in specific scenarios and regarding some functional characteristics.

Our assessment methodology is based on the QUEST test with some specific and local changes to adapt the questionnaire to our population and product. The designed questionnaire is similar to QUEST regarding device aspects such as usefulness, training, robustness, safety, dimensions, simplicity of use, appearance, and effort of installation. However, we could not be asked about service components for the prototype considered. Besides, we must have in mind that we are required to assess and to facilitate the robot improvement.

Another difference from QUEST is that a previous study is performed to obtain information to develop the final experiment according to users' needs expressed previously. In this pilot test information about each robot's interface is received, its ease of use, and which the best scenario is to carry out the experience. Following the users' priority, the scenario selected in our case is the bathroom.

In this line, the originality of our methodology lies in the interactivity and user-directed approach to assess satisfaction with assistive technology. With this approach, it is the user who determines the degree of importance of the variables that represent those factors more likely to influence the usability and the aspects to take into account to modify an existing prototype [6].

Three different functions have been tested in the bathroom scenario set in the hospital context: drinking a glass of water, brushing one's teeth, and washing one's face. From the experimental results obtained, we can conclude that the use of the prototype is correct for the functions of drinking a glass of water and brushing one's teeth. Its use for washing one's face with a sponge should be improved. Only two users agreed to test this last function, which may justify the low score obtained. 
In relation to the device characteristics, the best results are for its robustness, its multi-use functionality, and its speed of response. Lower scores are obtained for its dimensions and appearance. Although these results are good in average, some suggestions of the users to make the robot more useful focus on a smaller size of the prototype, the improvement of its mobility, voice recognition, the possibility of including additional tasks adding other terminal devices, and more safety conditions as far as the stopping function of the robot is concerned.

In relation to the overall assessment of the robot after its use, the patients have been asked two questions: one concerning the independence the robot could provide and the other one about replacing their career with the robot, freeing that person from doing the work. For the first question the average is 5 out of 10 and for the second one is 5.8 .

One patient have scored both items with 0 because his injury level, C7-C8, is less serious and he has an acceptable amount of mobility in his upper extremities. It is more efficient for him to do those functions by himself than with the robot's support. This fact has brought the average scores down quite a bit. Considering only the other four users, the averages would be 6.25 and 7.25 out of 10 , respectively. Anyway, the score is above 5, which can be considered to be a positive result in the overall assessment.

We have also found a relation between the level of injury and the final score. For users with lower levels of injury, C7-C8, the results are worse. It is due to the fact that their upper limb functional situation is better and they do not need such a sophisticated device to assist them. However, for users with greater mobility problems, the scores are higher, since they really take advantage of the robot assistance to support their daily activities.

There are other tests and results in literature in relation to specific population such as geriatric patients [30] or those with rheumatic disease [31]. Our case study is specifically directed to spinal cord injury population.

Considering devices themselves, it is possible to find some research about functional assessment for wheelchair users. Different tools to assess wheelchair functional behaviours have been described [32] [33]. In the same line, our experience reported here takes into account a specific device for a specific population with different spinal cord injury levels. 
Although a similar experience is reported by Rumau et al. [2] regarding a priori evaluation of the acceptance of an activity monitoring device for disabled elderly, only professional carer are asked instead of users.

It is necessary to state that our experience aims to give response to a specific problem involving disabled people and researchers who are far from the users and their environments. That can justify some limitations in the scientific methodology, in spite of the efforts to take all the practical aspects into account. However, we consider this experience very interesting and of great utility for all the parts involved in this research: the users, the ASIBOT team, and the clinical team.

\section{CONCLUSIONS}

According to the results presented in this paper, in general terms we can conclude that users must participate in both research and development processes of technical aids in order to focus the research objectives on specific needs and tools to make the end product more suitable and usable.

The methodology presented here proves its efficiency for the specific users, prototype, and scenario. In a systematic and structured way, it gathers the opinions of a group of users regarding a prototype and presents suggestions for improvement based on real demands.

\section{ACKNOWLEDGEMENTS}

The funding for this research was provided by the IMSERSO Nº3/05 (Social Service National Institute).

\section{DECLARATION OF INTEREST}

The authors report no declaration of interest. 


\section{REFERENCES}

[1] Harris A, Pinnington LL, Ward CD. Evaluating the impact of mobility-related assistive technology on the lives of disabled people: A review of outcome measures. British Journal of Occupational Therapy 2005; 553558.

[2] Rumeau P, Rialle V, Noury N. A priori evaluation of acceptance of an activity monitoring device for the disabled elderly using the HIS as a model. Smart Homes and Beyond, IOS Press 2006; pp. 130-137.

[3] Smith RO. Measuring the Outcomes of Assistive Technology: Challenge and Innovation. Journal of Assistive Technology 1996; 8:71-81.

[4] Fuhrer MJ. Assistive technology outcomes research: Challenges met and yet unmet. American Journal of Physical Medicine \& Rehabilitation 2001; 80:528-535.

[5] Jutai JW, Fuhrer MJ, Demers L, Scherer MJ, DeRuyter F. Toward a taxonomy of assistive technology device outcomes. American Journal of Physical Medicine \& Rehabilitation 2005; 84:294-302.

[6] Demers L, Weiss-Lambrou R, Ska B. Development of Quebec user evaluation of satisfaction with assistive technology (QUEST). Journal of Assistive Technology 1996; 8:3-13.

[7] Hochberg LR, Serruya MD, Friehs GM, Mukand JA, Saleh M, Branner A, Chen D, Penn RD, Donoghue JP. Neuronal ensemble control of prosthetic devices by a human with tetraplegia. Nature 2006; 442:164-171.

[8] Balaguer C, Giménez A, Jardón A. The MATS robot: Service climbing robot for personal assistance. IEEE Robotics \& Automation Magazine 2006; 13:51-58.

[9] Jardón A, Jiménez A, Correal R, Cabas R, Martínez S, Balaguer C. A portable light-weight climbing robot for personal assistance applications. Industrial Robot: An International Journal 2006; 33:303-307. 
[10] Balaguer C, Giménez A, Jardón A, Correal R, Martínez S. Proprio \& teleoperation of a robotic system for disabled persons assistance in domestic environments. In: Advances in telerobotics. Springer Tracts in Advanced Robotics (STAR); 2007. 31:389-402.

[11] Jardón A, Giménez A, Correal R, Martínez S, Balaguer C. ASIBOT: Robot portátil de asistencia a discapacitados. Concepto, arquitectura de control y evaluación clínica. Revista Iberoamericana de Automatica e Informatica Industrial 2008; 5(2):48-59.

[12] Balaguer C, Giménez A, Jardón A. Climbing robots' mobility for inspection and maintenance of 3D complex environments. Autonomous Robots 2005; 18(3):157-169.

[13] Jardón A. Assistive robot design methodology. Application to portable robot ASIBOT. Madrid: University Carlos III of Madrid; 2006.

[14] Cabas R, Balaguer C. Design and development of a light weight embodied robotic hand activated with only one actuator. IEEE/RSJ International Conference on Intelligent Robots and Systems (IROS'2005); 2005; Edmonton, Canada. 2005.

[15] Cabas R, Cabas LM, Jardón A, Balaguer C. Optimized design of the underactuated robotic hand. International Conference on Robotics and Automation (ICRA`06); 2006; Orlando, United States. 2006.

[16] Jardón A, Martinez S, Giménez A, Balaguer C. Assistive robots dependability in domestic environment: the ASIBOT kitchen test bed. 6th IARP/IEEE-RAS/EURON Workshop on Technical Challenges for Dependable Robots in Human Environments; 2008; Pasadena CA, USA. 2008.

[17] Demers L, Wessels R, Weiss-Lambrou R, Ska R, De Witte L. An international content validation of the Quebec user evaluation of satisfaction with assistive technology (QUEST). Occupational Therapy International 1999; 6:159-175. 
[18] Demers L, Ska B. Item analysis of the Quebec user evaluation of satisfaction with assistive technology (QUEST). Journal of Assistive Technology 2000; 12:96-105.

[19] Rubin J. Handbook of usability testing. How to plan, design and conduct effective tests. John Wiley \& sons, Inc. New York 1994; 57-78.

[20] Batavia AI, Hammer GS. Toward the development of consumer-based criteria for the evaluation of assistive device. Journal of Rehabililation, Research and Development 1990; 7:425-36.

[21] Lenkert JA, Paquet VL. A review of conceptual models for assistive technology outcomes research and practice. Journal of Assistive Technology 2003; 15:1-15.

[22] Virzi RA. Refining the test phase of usability evaluation: How many subjects is enough? Human Factors $1992 ; 34: 457-468$.

[23] Nielsen J, Landauer TK. A mathematical model of the finding of usability problems. ACM INTERCHI'93 Conference, 1993 April 24-29; Amsterdam, The Netherlands. 1993. pp. 206-213.

[24] Lewis JR. Sample sizes for usability studies: Additional considerations. Human Factors 1994; 36:368-378.

[25] Lewis JR. Evaluation of procedures for adjusting problem-discovery rates estimated from small samples. International Journal of Human-Computer Interaction 2001; 13:445-479.

[26] Turner CW, Lewis JR, Nielsen J. Determining usability test sample size. International Encyclopedia of Ergonomics and Human Factors 2006; Second Edition, 3:3084-3088.

[27] Simon SE, Patrick A. Understanding and assessing consumer satisfaction in rehabilitation. Journal of Rehabilitation Outcomes Measurement 1997; 1:1-14. 
[28] Carr-Hill RA. The measurement of patient satisfaction. Journal of Public Health Medicine 1992; 14:236490.

[29] Demers L, Ska B, Wessels R, Weiss-Lambrou R. Reliability, validity, and applicability of the Quebec user evaluation of satisfaction with assistive technology (QUEST 2.0) for adults with multiple sclerosis. Disability and Rehabilitation 2002; 24:21-30.

[30] Parker MG, Thorslund M. The use of technical aids among community based elderly. American Journal of Occupational Therapy $1991 ; 45: 712-18$.

[31] Rogers JC, Holm MB. Assistive technology device use in patients with rheumatic disease: A literature review. American Journal of Occupational Therapy 1992; 46:120-27.

[32] Stanley RK, Stafford DJ, Rasch E, Rodgers MM. Development of a functional assessment measure for manual wheelchair users. Journal of Rehabilitation, Research and Development 2003; 40:301-308.

[33] Kirby RL, Swuste J, Dupuis DJ, MacLeod DA, Monroe R. The wheelchair skills test: A pilot study of a new outcome measure. Archives of Physical Medicine and Rehabilitation 2002; 83:10-18. 
TABLES

\begin{tabular}{|l|c|c|c|c|c|c|c|}
\hline & C3-C4 & C4 & C5-C6 & C6 & C7-C8 & Total score & Average \\
\hline Helpful for user's autonomy & 1 & 1 & 2 & 1 & -1 & 4 & 0.8 \\
\hline Practical and useful & 1 & 1 & 2 & 2 & 0 & 6 & 1.2 \\
\hline Additional need of support & & & & & & & \\
\hline while using the robot & -1 & 0 & 1 & 1 & -1 & 0 & 0 \\
\hline Independency & 1 & 1 & 2 & 2 & 1 & 7 & 1.4 \\
\hline Physical effort & 2 & 1 & 2 & 2 & 0 & 7 & 1.4 \\
\hline Success & 1 & 0 & 2 & 2 & -1 & 4 & 0.8 \\
\hline Motivation & 1 & 1 & 2 & 1 & -1 & 4 & 0.8 \\
\hline Total score & 6 & 5 & 13 & 11 & -3 & 32 & \\
\hline Average & 0.86 & 0.71 & 1.86 & 1.57 & -0.43 & 0.91 & 0.91 \\
\hline
\end{tabular}

Table 1. Drinking function assessment. 


\begin{tabular}{|l|c|c|c|c|c|c|c|}
\hline & C3-C4 & C4 & C5-C6 & C6 & C7-C8 & Total score & Average \\
\hline Helpful for user's autonomy & 1 & 1 & 1 & 1 & -1 & 3 & 0.6 \\
\hline Practical and useful & 1 & 1 & 0 & 1 & -1 & 2 & 0.4 \\
\hline Additional need of support & & & & & & & \\
\hline while using the robot & -1 & 0 & -1 & 1 & 1 & 0 & 0 \\
\hline Independency & 1 & 0 & 0 & 2 & 0 & 3 & 0.6 \\
\hline Physical effort & 2 & 1 & 0 & 2 & 0 & 5 & 1 \\
\hline Success & 0 & -1 & -1 & 1 & 1 & 0 & 0 \\
\hline Motivation & 1 & 1 & -1 & 1 & 2 & 4 & 0.8 \\
\hline Total score & 5 & 3 & -2 & 9 & 2 & 17 & \\
\hline Average & 0.71 & 0.43 & -0.29 & 1.29 & 0.29 & 0.49 & 0.49 \\
\hline
\end{tabular}

Table 2. Brushing one's teeth function assessment. 


\begin{tabular}{|l|c|c|c|c|}
\hline & C5-C6 & C7-C8 & Total score & Average \\
\hline Helpful for user's autonomy & -1 & 0 & -1 & -0.5 \\
\hline Practical and useful & -1 & 0 & -1 & -0.5 \\
\hline Additional need of support & & & & \\
while using the robot & -2 & 1 & -1 & -0.5 \\
\hline Independency & -2 & 0 & -2 & -1 \\
\hline Physical effort & 0 & 0 & 0 & 0 \\
\hline Success & -2 & -1 & -3 & -1.5 \\
\hline Motivation & -2 & -2 & -4 & -2 \\
\hline Total score & -10 & -2 & -12 & \\
\hline Average & -1.43 & -0.29 & -0.86 & -0.86 \\
\hline
\end{tabular}

Table 3. Washing one's face function assessment. 


\begin{tabular}{|l|c|c|c|c|c|c|c|}
\hline & C3-C4 & C4 & C5-C6 & C6 & C7-C8 & Total score & Average \\
\hline Appearance & -1 & -1 & 0 & 1 & 0 & -1 & -0.2 \\
\hline Dimensions & -1 & -1 & -2 & 1 & -1 & -4 & -0.8 \\
\hline Usability & 1 & -1 & 2 & 1 & -2 & 1 & 0.2 \\
\hline Speed of response & 1 & 1 & -1 & 2 & 1 & 4 & 0.8 \\
\hline Safety & 0 & 0 & 1 & 2 & -1 & 2 & 0.4 \\
\hline Robustness & 2 & 1 & 1 & 2 & -1 & 5 & 1 \\
\hline Multi-use functionality & 1 & 2 & 1 & 2 & -1 & 5 & 1 \\
\hline Training & 2 & 2 & 0 & 0 & -1 & 3 & 0.6 \\
\hline Total score & 5 & 3 & 2 & 11 & -6 & 15 & 3 \\
\hline Average & 0.63 & 0.38 & 0.25 & 1.38 & -0.75 & 0.38 & 0.38 \\
\hline
\end{tabular}

Table 4. ASIBOT's characteristics assessment. 


\begin{tabular}{|l|c|c|c|c|c|c|c|}
\hline & C3-C4 & C4 & C5-C6 & C6 & C7-C8 & Average & users \\
\hline Independence provided by the & 8 & 6 & 5 & 6 & 0 & 5 & 6.25 \\
\hline robot in everyday live & 9 & 6 & 8 & 6 & 0 & 5.8 & 7.25 \\
\hline Replacing their carer with the & & & & & & & \\
\hline
\end{tabular}

Table 5. Overall assessment. 
FIGURES

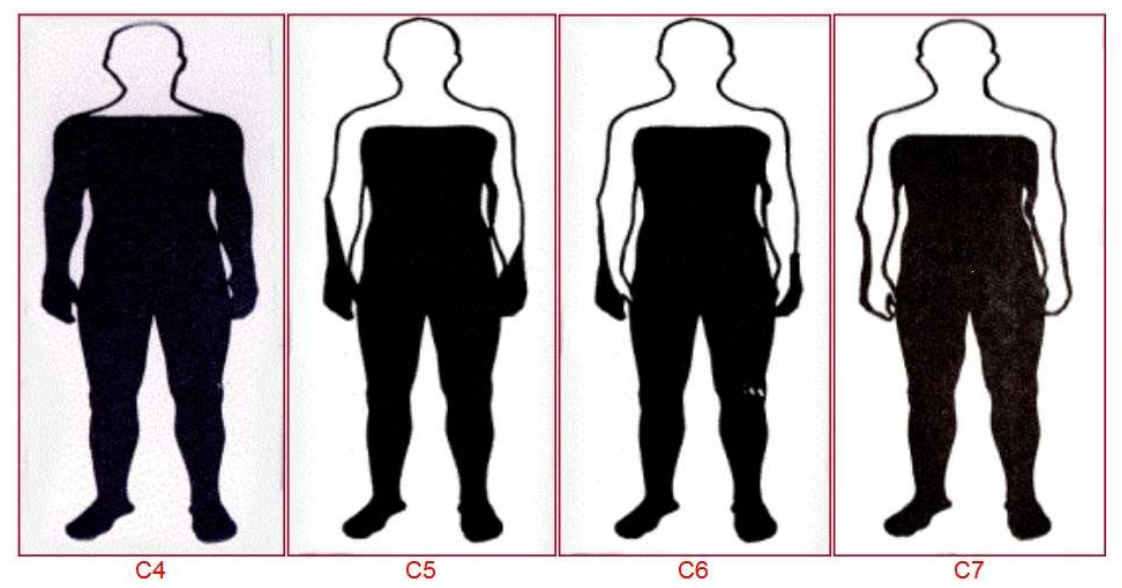

Figure 1. Levels of spinal cord injury.

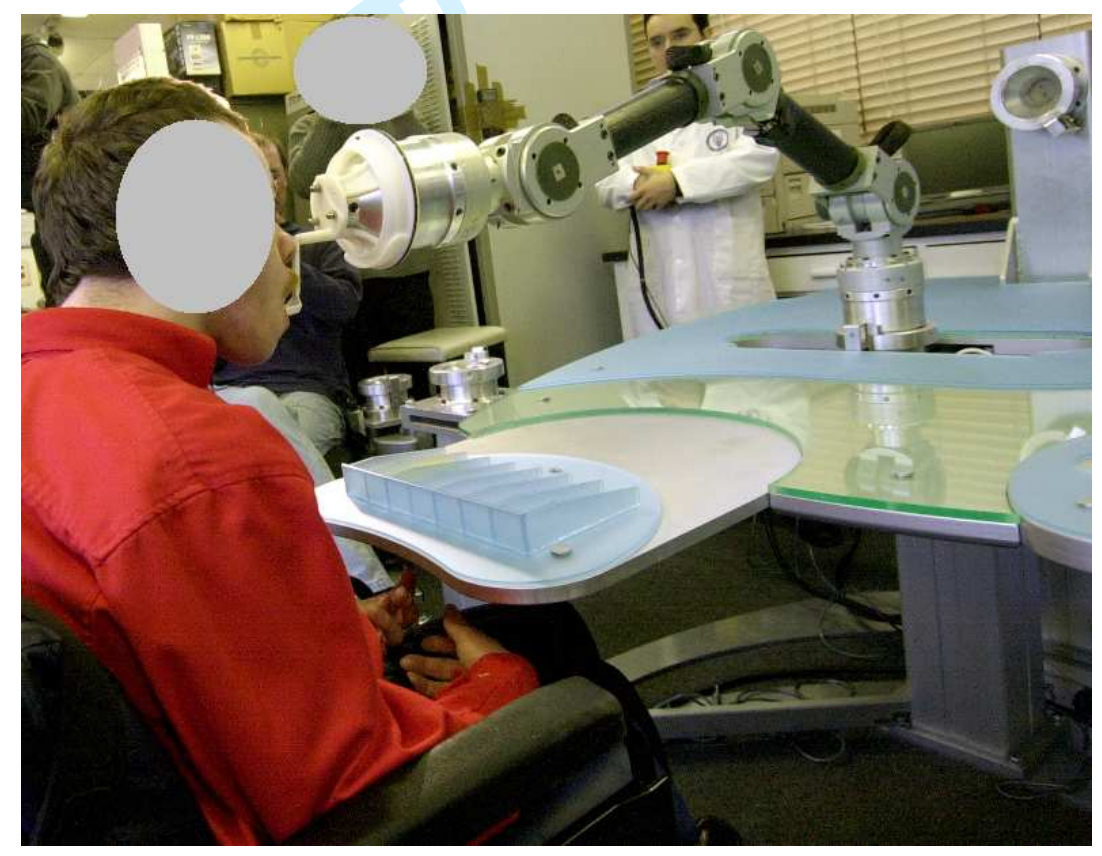

Figure 2. Robot image in a feeding operation. 


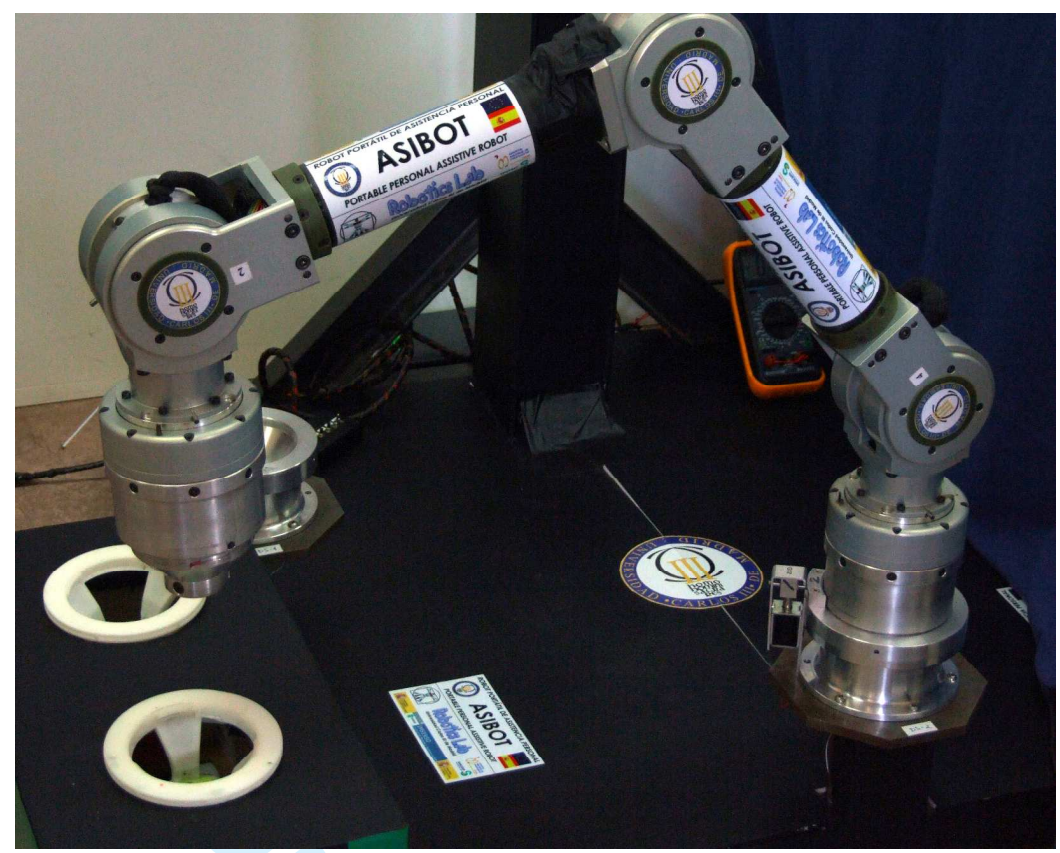

Figure 3. ASIBOT performing docking in both tips' connectors.

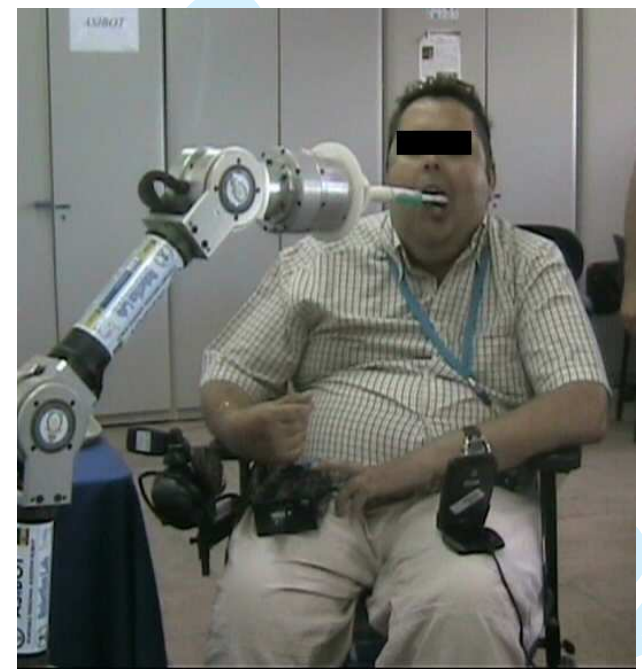

Fig. 4. ASIBOT helping in a teeth brushing task. 

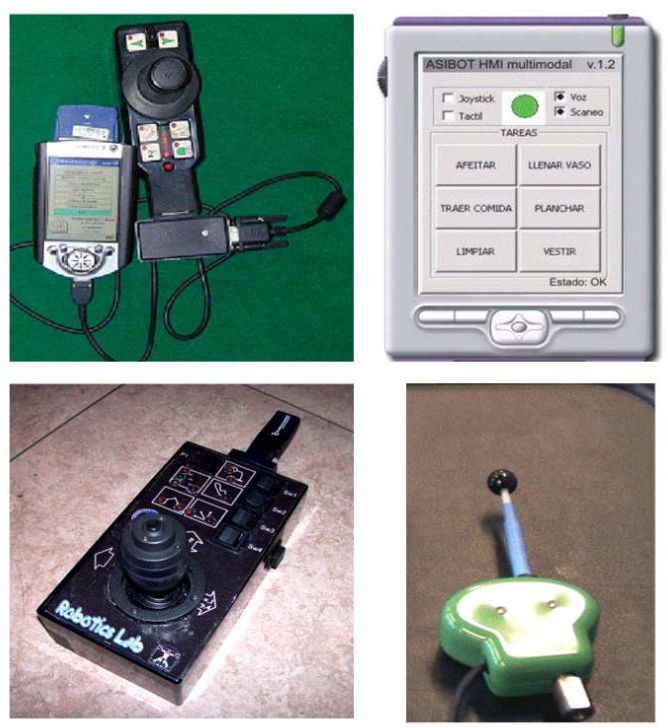

Figure 5. PDA screen details and access-granted devices.

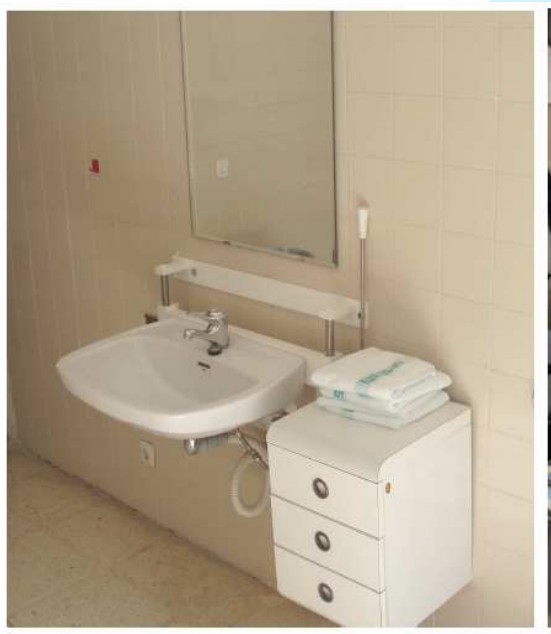

(a)

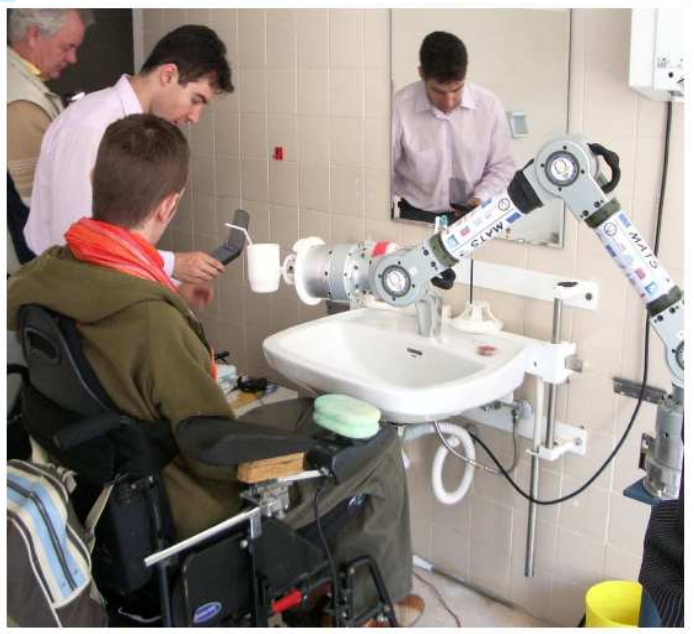

(b)

Figure 6. Bathroom scenario: before (a) and after (b) adaptations. 


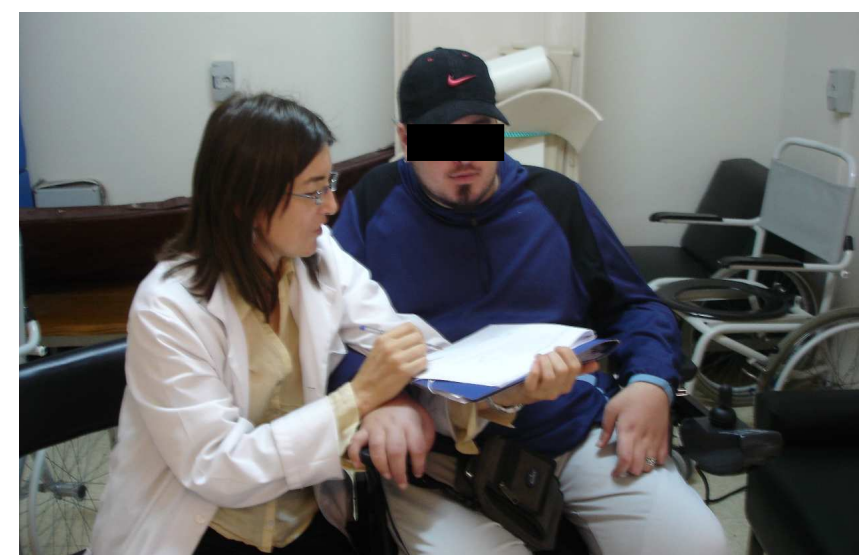

Figure 7. Occupational therapist gathering user's perception.

\section{FIGURE CAPTIONS}

Figure 1. Levels of spinal cord injury.

Figure 2. Robot image in a feeding operation.

Figure 3. ASIBOT performing docking in both tips' connectors.

Figure 4. ASIBOT helping in a teeth brushing task.

Figure 5. PDA screen details and access-granted devices.

Figure 6. Bathroom scenario: before (a) and after (b) adaptations.

Figure 7. Occupational therapist gathering user's perception. 


\section{FIGURE CAPTIONS}

Figure 1. Levels of spinal cord injury.

Figure 2. Robot image in a feeding operation.

Figure 3. ASIBOT performing docking in both tips' connectors.

Figure 4. ASIBOT helping in a teeth brushing task.

Figure 5. PDA screen details and access-granted devices.

Figure 6. Bathroom scenario: before (a) and after (b) adaptations.

Figure 7. Occupational therapist gathering user's perception.

\section{TABLE CAPTIONS}

Table 1. Drinking function assessment.

Table 2. Brushing one's teeth function assessment.

Table 3. Washing one's face function assessment.

Table 4. ASIBOT's characteristics assessment.

Table 5. Overall assessment. 